\title{
Influence of Different Drying Techniques on Phenolic Compounds, Antioxidant Capacity and Colour of Ziziphus jujube Mill. Fruits
}

\author{
Aneta Wojdyło ${ }^{1, * \mathbb{C}}$, Krzysztof Lech $^{2}$, Paulina Nowicka ${ }^{1}$, Francisca Hernandez ${ }^{3}{ }^{\mathbb{D}}$, \\ Adam Figiel ${ }^{2}\left(\mathbb{D}\right.$ ) and Angel Antonio Carbonell-Barrachina ${ }^{4}(\mathbb{D})$ \\ 1 Department of Fruit, Vegetable and Plant Nutraceutical Technology, Wrocław University of Environmental \\ and Life Sciences, 37 Chełmońskiego Street, 51-630 Wroclaw, Poland \\ 2 Institute of Agricultural Engineering, Wrocław University of Environmental and Life Sciences, \\ 37/41 Chełmońskiego Street, 51630 Wrocław, Poland \\ 3 Department of Plant Sciences and Microbiology, Research Group "Plant Production and Technology", \\ Escuela Politécnica Superior de Orihuela, Universidad Miguel Hernández de Elche, Ctra. de Beniel, km 3.2, \\ 03312 Orihuela, Alicante, Spain \\ 4 Department of Agro-Food Technology, Research Group "Food Quality and Safety”, Escuela Politécnica \\ Superior de Orihuela (EPSO), Universidad Miguel Hernández de Elche, Ctra. de Beniel, km 3.2, \\ 03312 Orihuela, Alicante, Spain \\ * Correspondence: aneta.wojdylo@upwr.edu.pl; Tel.: +48-71-320-7706
}

Received: 1 May 2019; Accepted: 20 June 2019; Published: 26 June 2019

\begin{abstract}
This study was to present the effect of different parameters of combined methods of drying such as vacuum-microwave (VMD: 480, $120 \mathrm{~W}$ ), hot air (CDD: 70, 60, 50 ${ }^{\circ} \mathrm{C}$ ) and combined methods as pre-drying by CD and finish drying by VMD (CD-VMD: $60^{\circ} \mathrm{C}+480 / 120 \mathrm{~W}$ ) in order to avoid a rapid increase in temperature at the critical moisture content of ca. $1 \mathrm{~kg} / \mathrm{kg} \mathrm{dm}$ (dry mass). Control samples were prepared by freeze-drying (FD). Drying kinetics, including the temperature profile of dried material, as well as on some quality factors of the finished product as phenolic compounds, antioxidant capacity, and color were evaluated. The increase in air temperature during $\mathrm{CD}$ as well as the increase in material temperature during VMD deteriorated dried product quality in terms of the content of phenolic compounds, antioxidant activity and color. Dried jujube fruits have a long shelf life and therefore may be a fine alternative to fresh fruit all year round.
\end{abstract}

Keywords: jujube; microwave power levels; air temperature; bioactive compounds

\section{Introduction}

Nowadays dried fruits and vegetablesare highly popular valuable healthy snacks. Drying affects the fruit appearance and chemical composition but it allows for effective handling of raw materials and prolonging their shelf life as it inhibits enzymatic degradation and limits microbial growth [1].

Selection of an adequate drying method and its parameters yields a product with high antioxidant activity, only slightly changed in appearance as compared to fresh fruit, and with a more favorable taste. Considering consumer preferences, appropriate drying method should be selected, so as to retain maximum levels of bioactive compounds in the final product. Dried fruit snacks are good sources of dietary fibre, minerals, vitamins, and bioactive compounds. Their antioxidant properties are due mainly to the presence of carotenoids, phytosterols, phenolic compounds and vitamins $C$ and $E$ [2].

Many reports claim that choosing right parameters of the drying method is as important as choosing the method itself [3-6]. Currently, one of the most popular drying metod is microwave drying, because contrary to hot air drying method, it reduces the drying time of plant materials 
without any meaningful decline of quality. At an industrial level, food processing using this technique has been reported to be both cost effective and feasible [7]. Systems of drying combining (such as: microwave and hot air drying) not only increase drying rates but also responsible for quality of the dry products [8-10]. Nowadays, day by day microwave drying techniques is widely used in combination with pre-drying by hot air-drying systems which removes free water from the product surface, and finished by microwave when energy from microwave removes water from inside the product [6,11].

Jujube (Ziziphus jujuba Mill.) fruits are highly favored by consumers, as they are tasty and rich in nutrients, especially vitamins, minerals, and polyphenols [12-14]. The fruits are a good source of natural antioxidant compounds, namely polyphenols that confer numerous health benefits i.e. show antiobesity antiproliferative, antitumor, antioxidant, antiinflamatory, and proapoptopic properties, and may protect against cardiovascular diseases and type II diabetes [15]. Generally, jujube fruits are eaten fresh, however, their shelf-life is short ( $2-4$ days at ambient temperature) and their rapid decay is problematic for postharvest management and advance processing [16,17].

Therefore, the aim of this present study was to determine the effects of different drying methods, such as vacuum-microwave drying (VMD) at different powers (120 and $480 \mathrm{~W})$, hot air drying (CD) at different temperatures $\left(50,60,70^{\circ} \mathrm{C}\right)$, and hot air pre-drying followed by vacuum-microwave finish drying (CD-VMD) on the quality of three different jujube cultivars ('GAL', 'MSI', and 'PSI'). In obtain sample it was evaluated drying kinetics, including temperature profile of the dried material, and the quality of the dried products, including color, total phenolic compounds (TPC), and antioxidant capacity (ORAC). Finally, freeze drying (FD) was used as a reference or control method, as it provides high quality of the final products.

\section{Results and Discussion}

\subsection{Drying Kinetics}

Figures 1-3 show drying kinetics of jujube fruits as a function of MR change over time. Preliminary tests identified modified Page model as the best one describing the drying kinetics (Equation (1)):

$$
M R=A \cdot e^{-k \cdot t^{n}}
$$

where $A, n, k$ and $t$ are constants and drying time, respectively.

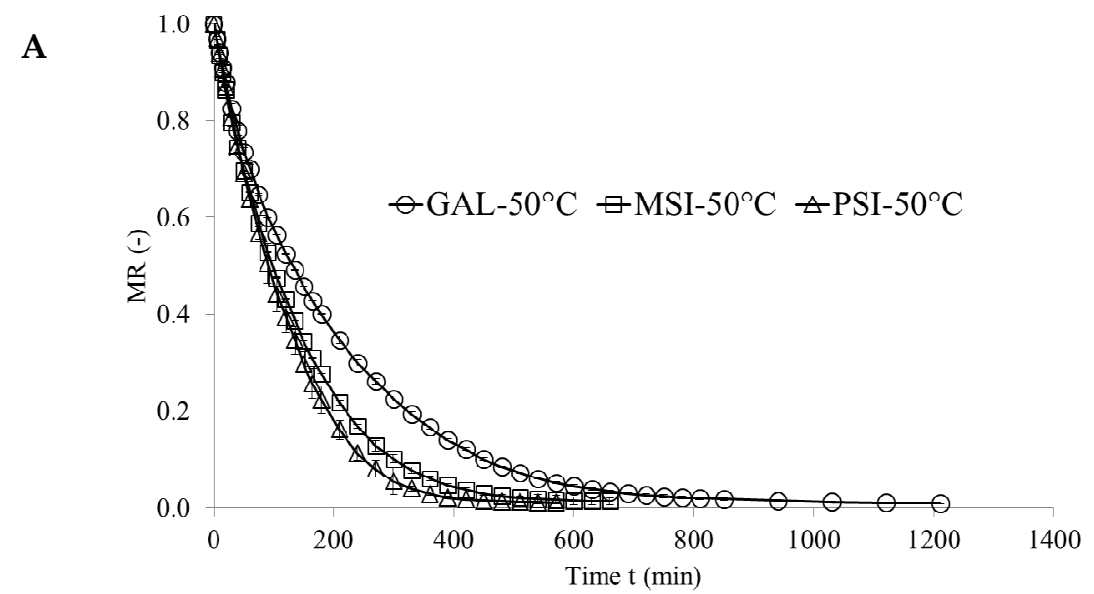

Figure 1. Cont. 
B

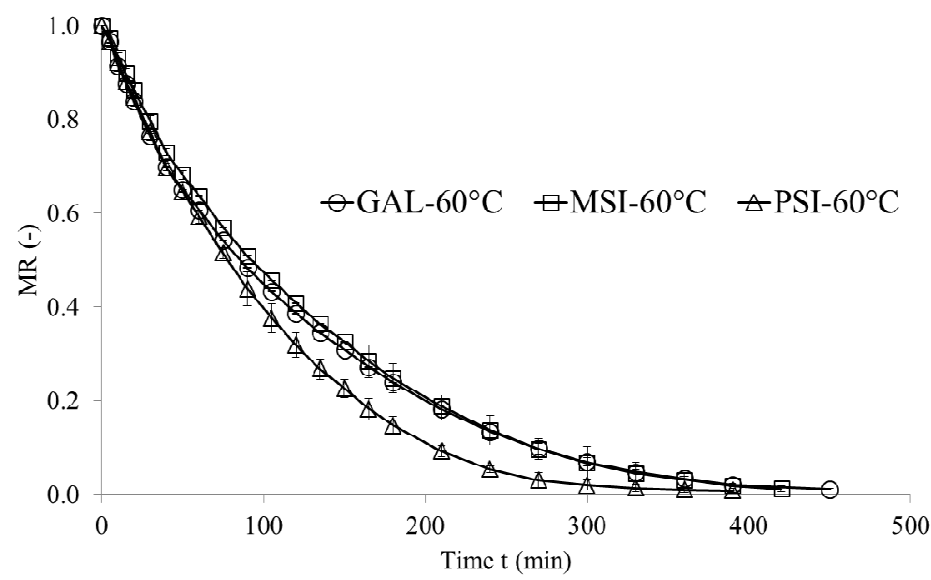

C

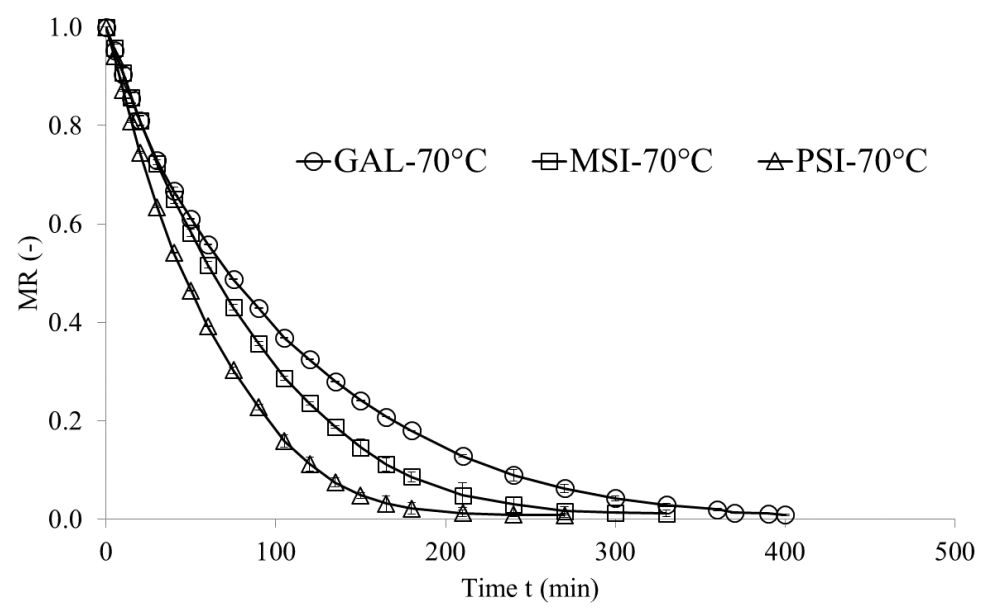

Figure 1. (A) Drying kinetics of jujube fruits during $\mathrm{CD} 50^{\circ} \mathrm{C}$; (B) Drying kinetics of jujube fruits during $\mathrm{CD} 60^{\circ} \mathrm{C}$; (C) Drying kinetics of jujube fruits during $\mathrm{CD} 70{ }^{\circ} \mathrm{C}$.

A

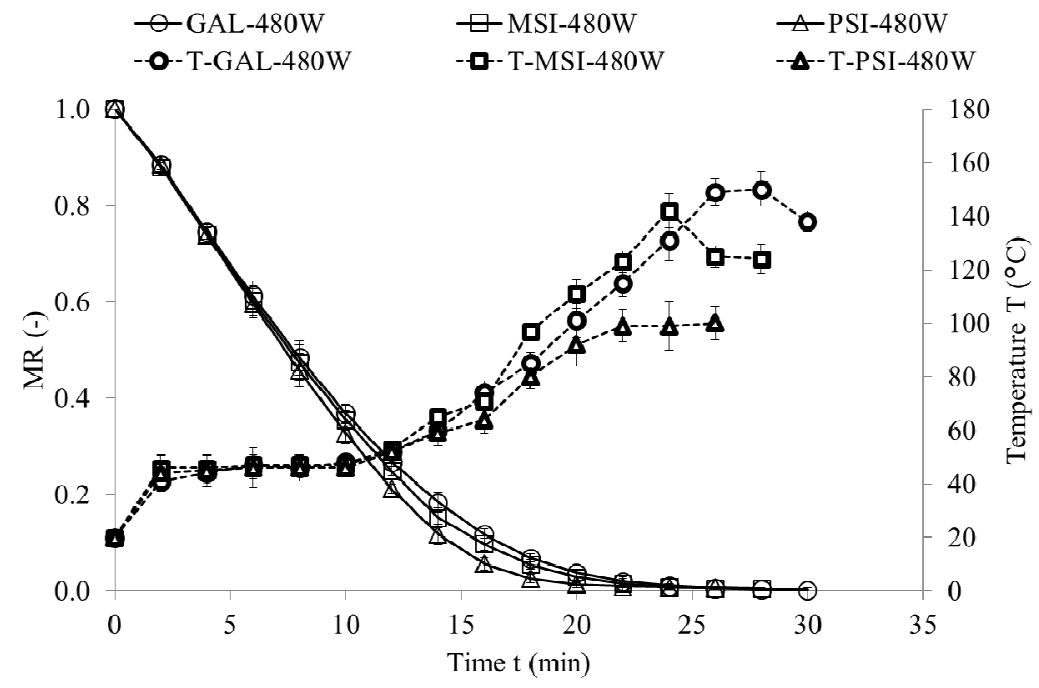

Figure 2. Cont. 
B

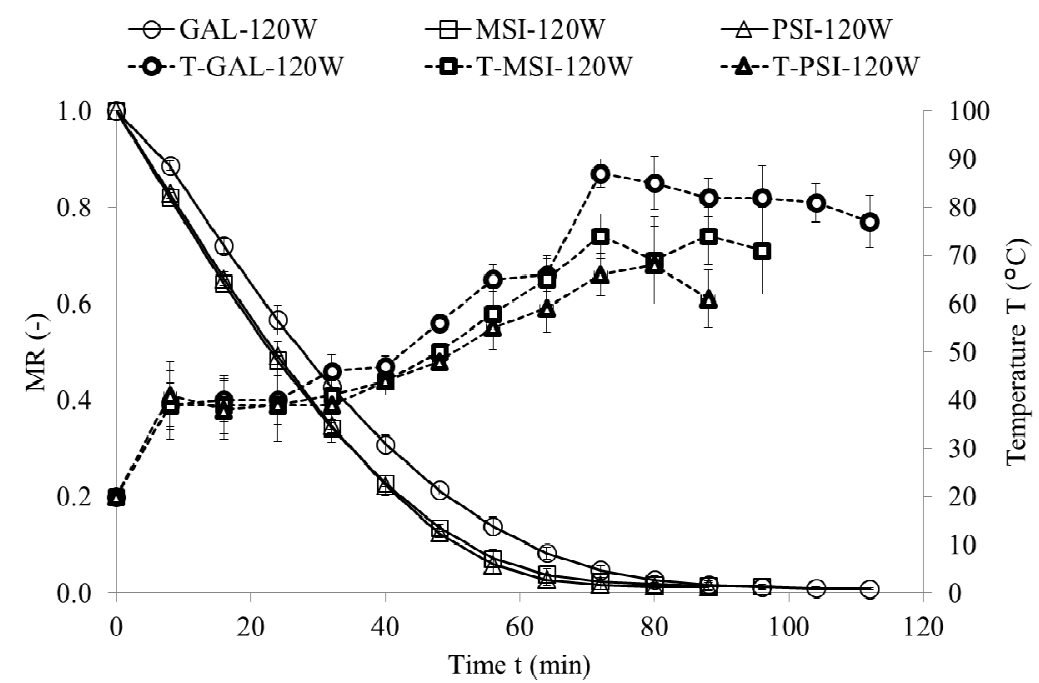

C

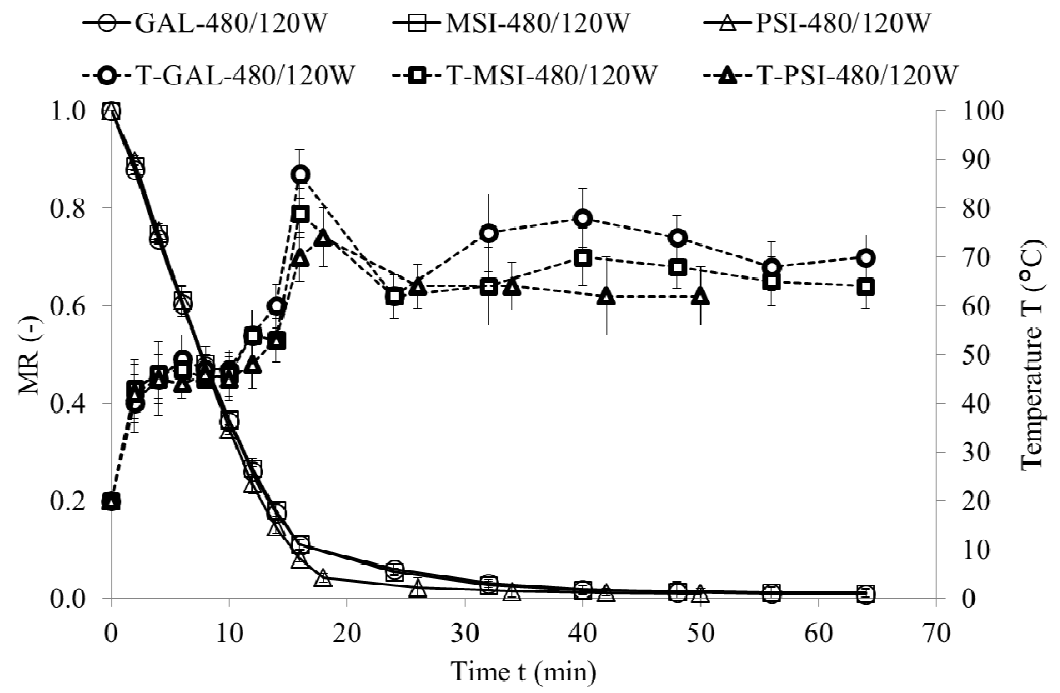

Figure 2. (A) Drying kinetics of jujube fruits during VMD $480 \mathrm{~W}$; (B) Drying kinetics of jujube fruits during VMD 120 W; (C) Drying kinetics of jujube fruits during VMD 480/120 W.

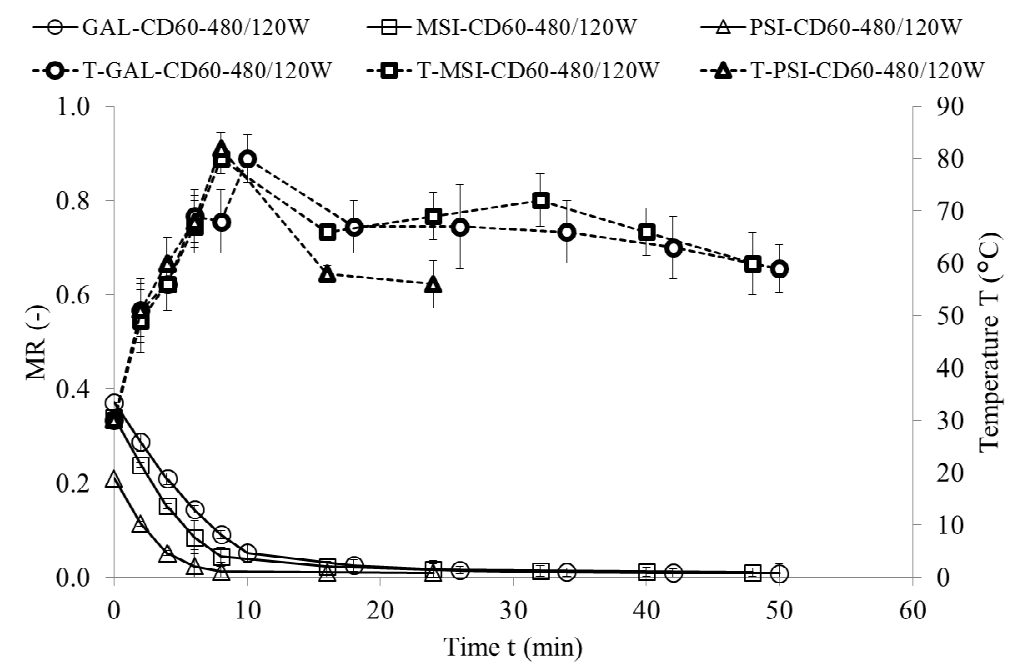

Figure 3. Drying kinetics of jujube fruits during pre-drying by $\mathrm{CD}\left(60^{\circ} \mathrm{C}\right)$ and finished by VMD $(480 / 120 W)$. 
This model was used before to characterize the drying kinetics in jujube [16], chokeberry [17] and plum [18]. Table 1 presents the model constants, coefficients of determinations $\left(R^{2}\right)$, root mean square error (RMSE), maximum temperature and final moisture content and drying time. The values of RMSE below 0.0181 and of $R^{2}$ above 0.9879 demonstrate very good fit of the model to the empirical data [19]. Parameter A represents MR value at the beginning of the drying, which equals 1 for $C D$ and VMD. For CD-VMD method this variable reaches MR after hot air pre-drying. Similar results were obtained for drying of pomegranate arils [20]. Parameters $n$ and $k$ determine the drying rate-the greater they are, the shorter the drying time [17]. In all cultivars they were significantly higher in VMD variant than in CD. Materials with high water content heat up faster during microwave drying as they absorb more microwave power. This considerably improves the drying time [21]. Additionally, reducing pressure increases the pressure gradient and makes the drying process up to five times faster [7]. The shortest drying time (26 $\mathrm{min}$ ) was achieved for cv. 'PSI' dried by VMD at $480 \mathrm{~W}$, and the longest (1210 $\mathrm{min}$ ) for cv. 'GAL' exposed to hot air drying at $50^{\circ} \mathrm{C}$. 'PSI' fruits featured the highest initial moisture content $\left(4.72 \mathrm{~kg} \cdot \mathrm{kg}^{-1} \mathrm{~d} . \mathrm{w}.\right)$, but their drying time was shorter and maximum sample temperature during VMD was lower than in other cultivars ('GAL' and 'MSI'). This might be due to how water is bound by cellular layout and structure in individual cultivars (cv. 'PSI' had higher cell density than 'GAL', data not present), and to plant defense mechanisms against water loss [22]. Final moisture content of dried fruits reported by [16] was below $5.66 \%$, and this corroborated our results for jujube fruits (Table 1.)

\subsection{Energy Consumption}

Figure 4A-C show specific energy consumption profiles depending on material moisture content during CD (Figure 4A), VMD (Figure 4B) and CD-VMD (Figure 4C). The specific energy consumption is expressed in $\mathrm{kJ} \cdot \mathrm{g}^{-1}$ fresh weight (f.w.). We observed a rapid growth in energy consumption in materials with low moisture content (below $0.25 \mathrm{~kg} \cdot \mathrm{kg}^{-1} \mathrm{~d}$.w.), that is at the end of the drying, when the process slows down as water is removed from inside the material (internal diffusion). Similar energy consumption profiles were reported for garlic [9] and pomegranate [8]. Table 1 presents total specific energy consumption expressed in $\mathrm{kJ} \cdot \mathrm{g}^{-1} \mathrm{f} . \mathrm{w}$. and $\mathrm{kJ} \cdot \mathrm{g}^{-1}$ water. The variable was the lowest during VMD at $480 \mathrm{~W}\left(21.5 \mathrm{~kJ} \cdot \mathrm{g}^{-1} \mathrm{f} . \mathrm{w} ., 26.27 \mathrm{~kJ} \cdot \mathrm{g}^{-1}\right.$ water) for $\mathrm{cv}$. 'PSI', and the highest during $\mathrm{CD}$ at $50{ }^{\circ} \mathrm{C}$ (166.56 kJ.g ${ }^{-1}$ f.w., $205.77 \mathrm{~kJ} \cdot \mathrm{g}^{-1}$ water) for cv. 'GAL'. Increased air temperature during CD and higher microwave power during VMD resulted in lower energy consumption. Similar conclusions were drawn following hot air drying of pomegranate fruits [23] and microwave drying of parsley leaves [24]. Combined drying (CD-VMD) reduced energy consumption by over 1.5 times as compared with CD. The same was reported by Jiang et al. [25] who experimented with drying okra. 
Table 1. Drying time, maximum temperature of the samples, final moisture content, final specific energy consumption, cumulative energy efficiency and constants of the models describing the drying kinetics of jujube fruits.

\begin{tabular}{|c|c|c|c|c|c|c|c|c|c|c|c|c|c|}
\hline \multirow[t]{2}{*}{ Cultivars } & \multirow{2}{*}{$\begin{array}{l}\text { Drying } \\
\text { Conditions }\end{array}$} & \multicolumn{3}{|c|}{ Constants } & \multicolumn{2}{|c|}{ Statistics } & \multicolumn{2}{|c|}{$\begin{array}{l}\text { Drying Time } \\
\text { (min) }\end{array}$} & \multirow{2}{*}{$\begin{array}{l}\operatorname{Tmax} \\
\left({ }^{\circ} \mathrm{C}\right)\end{array}$} & \multirow[t]{2}{*}{ Mcwb (\%) } & \multicolumn{2}{|c|}{$\begin{array}{l}\text { Final Specific Energy } \\
\text { Consumption }\end{array}$} & \multirow{2}{*}{$\begin{array}{l}\text { Cumulative } \\
\text { Energy } \\
\text { Efficiency }(\%)\end{array}$} \\
\hline & & $A$ & $k$ & $n$ & RMSE & $R^{2}$ & CD & VMD & & & $\mathrm{kJ} \cdot \mathrm{g}^{-1} \mathrm{fw}$ & $\mathrm{kJ} \cdot \mathrm{g}^{-1}$ water & \\
\hline \multirow{7}{*}{ ‘GAL' } & VMD 480 & 1 & 0.0345 & 1.481 & 0.0133 & 0.9984 & - & 30 & $150 \pm 7 \mathrm{j}$ * & 0.95 & $24.76 \pm 1.29 \mathrm{ab}$ & $30.54 \pm 1.59 \mathrm{ab}$ & $7.73 \pm 0.22 \mathrm{k}$ \\
\hline & VMD 120 & 1 & 0.0054 & 1.465 & 0.007 & 0.9996 & - & 112 & $87 \pm 3 f$ & 3.54 & $50.74 \pm 2.64 \mathrm{f}$ & $63.58 \pm 3.31 \mathrm{f}$ & $3.71 \pm 0.13 f$ \\
\hline & VMD 480/120 & 1 & 0.0436 & 1.379 & 0.0167 & 0.9975 & - & 64 & $87 \pm 5 f g$ & 4.04 & $35.13 \pm 1.83 c$ & $43.34 \pm 2.25 c$ & $5.45 \pm 0.22 \mathrm{i}$ \\
\hline & CD-VMD 480/120 & 0.372 & 0.111 & 1.212 & 0.0103 & 0.9924 & 120 & 50 & $80 \pm 5$ defg & 3.75 & $46.68 \pm 2.43 \mathrm{ef}$ & $57.49 \pm 2.99 \mathrm{e}$ & $4.10 \pm 0.13 g$ \\
\hline & $\mathrm{CD} 50^{\circ} \mathrm{C}$ & 1 & 0.0076 & 0.930 & 0.008 & 0.9994 & 1210 & - & $50 \pm 2 a$ & 4.24 & $166.56 \pm 6.66 \mathrm{k}$ & $205.77 \pm 8.231$ & $1.16 \pm 0.04 a$ \\
\hline & $\mathrm{CD} 60^{\circ} \mathrm{C}$ & 1 & 0.0085 & 0.994 & 0.0137 & 0.9982 & 420 & - & $60 \pm 2 b$ & 4.30 & $75.52 \pm 3.02 \mathrm{i}$ & $94.92 \pm 3.8 \mathrm{i}$ & $2.51 \pm 0.08 \mathrm{~cd}$ \\
\hline & $\mathrm{CD} 70^{\circ} \mathrm{C}$ & 1 & 0.0102 & 0.989 & 0.0093 & 0.9992 & 450 & - & $70 \pm 2 c$ & 3.76 & $65.52 \pm 3.28 \mathrm{~h}$ & $103.32 \pm 4.65 j$ & $2.30 \pm 0.07 \mathrm{bc}$ \\
\hline \multirow{7}{*}{ ‘MSI' } & VMD 480 & 1 & 0.0342 & 1.506 & 0.0151 & 0.9979 & - & 28 & $142 \pm 7 i$ & 1.78 & $23.12 \pm 1.2 \mathrm{a}$ & $29.00 \pm 1.51 \mathrm{a}$ & $8.14 \pm 0.281$ \\
\hline & VMD 120 & 1 & 0.0098 & 1.368 & 0.0137 & 0.9982 & - & 96 & $74 \pm 5$ cde & 5.64 & $43.65 \pm 2.27 \mathrm{de}$ & $54.55 \pm 2.84 \mathrm{de}$ & $4.33 \pm 0.14 \mathrm{~g}$ \\
\hline & VMD 480/120 & 1 & 0.0404 & 1.406 & 0.0154 & 0.9979 & - & 64 & $79 \pm 5$ defg & 4.42 & $35.02 \pm 1.82 c$ & $44.21 \pm 2.3 c$ & $5.34 \pm 0.21 \mathrm{i}$ \\
\hline & CD-VMD 480/120 & 0.341 & 0.154 & 1.216 & 0.0127 & 0.9957 & 120 & 48 & $80 \pm 3$ defg & 4.20 & $45.02 \pm 2.34 \mathrm{e}$ & $56.67 \pm 2.95 \mathrm{e}$ & $4.16 \pm 0.13 g$ \\
\hline & $\mathrm{CD} 50^{\circ} \mathrm{C}$ & 1 & 0.0064 & 1.025 & 0.007 & 0.9996 & 660 & - & $50 \pm 2 a$ & 5.53 & $91.08 \pm 4.1 \mathrm{j}$ & $113.48 \pm 5.11 \mathrm{k}$ & $2.10 \pm 0.1 \mathrm{~b}$ \\
\hline & $\mathrm{CD} 60^{\circ} \mathrm{C}$ & 1 & 0.0057 & 1.066 & 0.0131 & 0.9984 & 420 & - & $60 \pm 2 b$ & 4.20 & $70.28 \pm 2.81 \mathrm{~h}$ & $90.29 \pm 3.61 \mathrm{hi}$ & $2.64 \pm 0.08 \mathrm{~d}$ \\
\hline & $\mathrm{CD} 70^{\circ} \mathrm{C}$ & 1 & 0.0067 & 1.126 & 0.0088 & 0.9993 & 330 & - & $70 \pm 2 c$ & 4.56 & $69.06 \pm 3.45 \mathrm{~h}$ & $87.12 \pm 4.36 \mathrm{~h}$ & $2.73 \pm 0.1 \mathrm{de}$ \\
\hline \multirow{7}{*}{ ‘PSI' } & VMD 480 & 1 & 0.0295 & 1.604 & 0.0181 & 0.9972 & - & 26 & $100 \pm 6 h$ & 2.74 & $21.50 \pm 1.12 \mathrm{a}$ & $26.27 \pm 1.37 \mathrm{a}$ & $8.98 \pm 0.3 m$ \\
\hline & VMD 120 & 1 & 0.0077 & 1.440 & 0.0171 & 0.9973 & - & 88 & $68 \pm 8 c$ & 5.66 & $39.84 \pm 2.07 \mathrm{~cd}$ & $48.89 \pm 2.54 \mathrm{~cd}$ & $4.83 \pm 0.15 h$ \\
\hline & VMD 480/120 & 1 & 0.0302 & 1.563 & 0.0146 & 0.9981 & - & 50 & $74 \pm 6 c d$ & 4.84 & $29.46 \pm 1.53 b$ & $36.02 \pm 1.87 \mathrm{~b}$ & $6.55 \pm 0.3 \mathrm{j}$ \\
\hline & CD-VMD 480/120 & 0.21 & 0.285 & 1.136 & 0.0072 & 0.9879 & 120 & 24 & $82 \pm 3$ efg & 4.17 & $39.13 \pm 2.93 \mathrm{~cd}$ & $47.66 \pm 3.57 c$ & $4.95 \pm 0.17 \mathrm{~h}$ \\
\hline & $\mathrm{CD} 50^{\circ} \mathrm{C}$ & 1 & 0.0045 & 1.121 & 0.0073 & 0.9996 & 570 & - & $50 \pm 2 a$ & 4.76 & $78.58 \pm 3.54 \mathrm{i}$ & $96.22 \pm 4.33 \mathrm{i}$ & $2.47 \pm 0.08 \mathrm{~cd}$ \\
\hline & $\mathrm{CD} 60^{\circ} \mathrm{C}$ & 1 & 0.005 & 1.141 & 0.0119 & 0.9988 & 390 & - & $60 \pm 2 b$ & 3.85 & $65.52 \pm 3.28 \mathrm{~h}$ & $79.71 \pm 3.99 \mathrm{~g}$ & $2.99 \pm 0.07 \mathrm{e}$ \\
\hline & $\mathrm{CD} 70^{\circ} \mathrm{C}$ & 1 & 0.0094 & 1.133 & 0.0105 & 0.9991 & 270 & - & $70 \pm 2 c$ & 3.60 & $56.73 \pm 2.84 \mathrm{~g}$ & $68.98 \pm 3.45 f$ & $3.45 \pm 0.09 \mathrm{f}$ \\
\hline
\end{tabular}

* Values followed by the same letter \pm standard deviation; within the same column, are not significantly different ( $p<0.05$, Duncan's multiple range test); FD-freeze drying; CD-convective

drying; VMD-vacuum-microwave drying; CD-VMD- convective-vacuum-microwave drying; $\mathrm{A}, \mathrm{k}$ and $\mathrm{n}$ are constants of the modified Page model; RMSE-mean square errors;

$R^{2}$-determination coefficient; Tmax-temperature maximal; Mcwb-moisture content wet basis; in each column different letters mean significant differences between samples. 

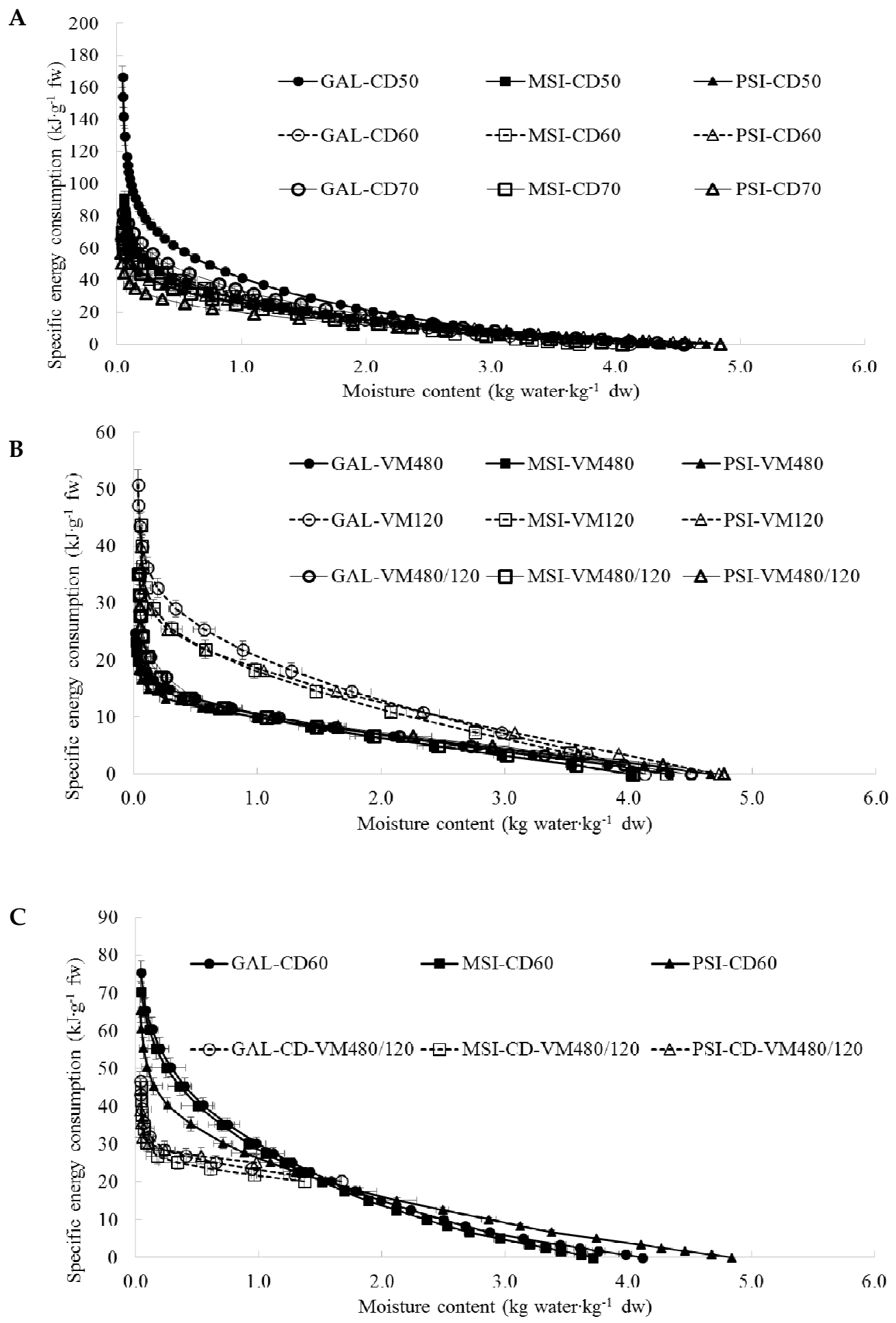

Figure 4. (A) Profiles of specific energy consumption during CD of jujube fruits. (B) Profiles of specific energy consumption during VMD of jujube fruits. (C) Profiles of specific energy consumption during $\mathrm{CD} 60^{\circ} \mathrm{C}$ and CD-VMFD $\left(60{ }^{\circ} \mathrm{C} 480 / 120 \mathrm{~W}\right)$ of jujube fruits.

Figure 5 shows cumulative energy efficiency profiles. The cumulative energy efficiency closely correlates with specific energy consumption-increased energy consumption considerably reduces drying efficiency [26]. Table 1 displays final cumulative energy efficiency. It was the highest during VMD at $480 \mathrm{~W}(8.98 \%)$ for cv. 'PSI' and the lowest during $\mathrm{CD}$ at $50{ }^{\circ} \mathrm{C}(1.16 \%)$ for cv. 'GAL'. 
A

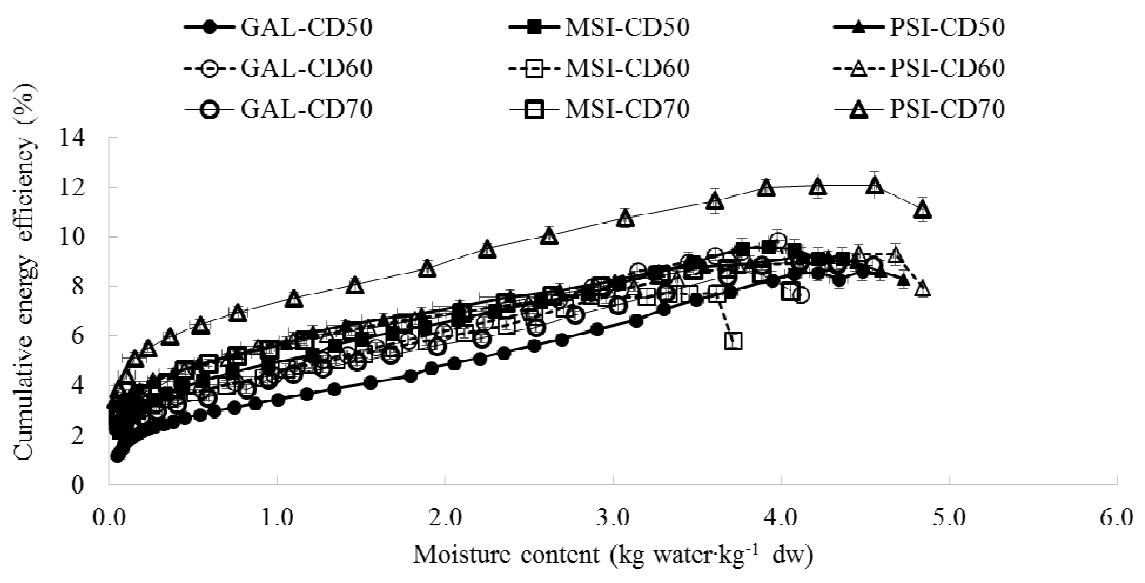

B

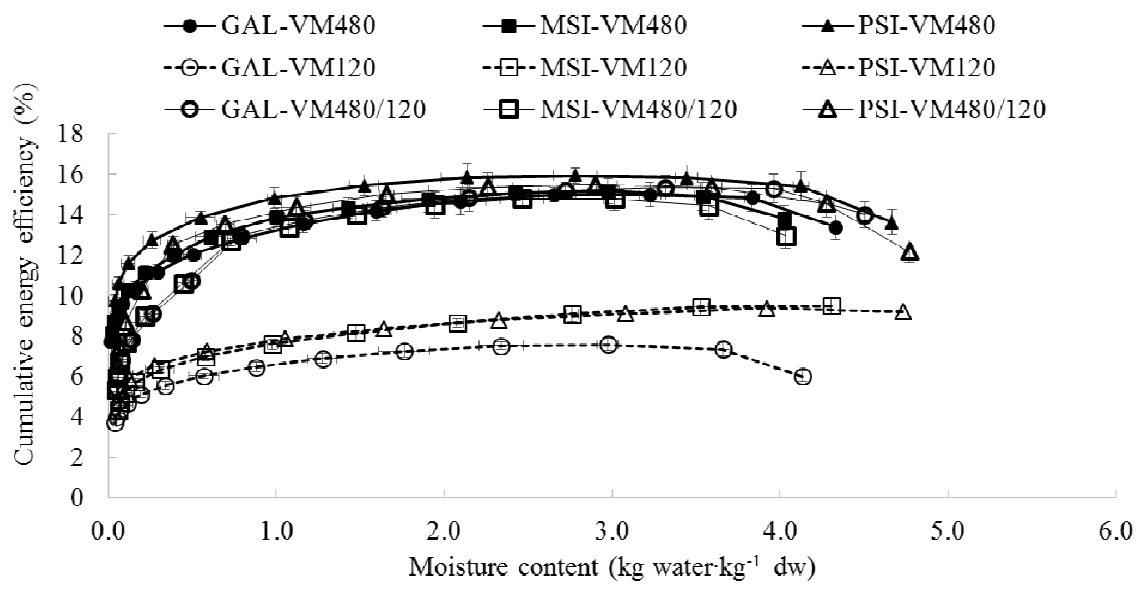

C

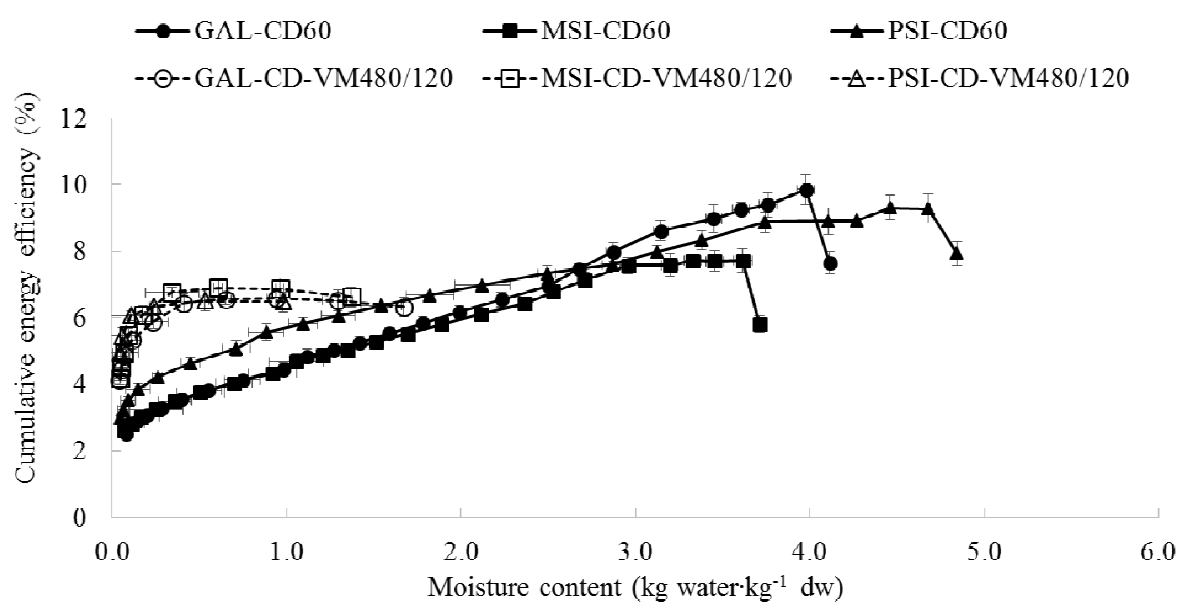

Figure 5. (A) Cumulative energy efficiency during $C D$ of jujube fruits. (B) Cumulative energy efficiency during VMD of jujube fruits. (C) Cumulative energy efficiency during CD $60^{\circ} \mathrm{C}$ and $\mathrm{CD}$-VMD $\left(60^{\circ} \mathrm{C}\right.$ $480 / 120 \mathrm{~W})$ of jujube fruits.

\subsection{Color}

Table 2 shows color parameters for the flesh of fresh and dried jujube fruits. $L^{*}, a^{*}$ and $b^{*}$ were highly similar in the fresh material for all three cultivars. Irrespective of drying method, $L^{*}$ was lower in dried than fresh fruits, which means the fruits darkened. Chen et al. [16] reported an increase in $L^{*}$ in jujube fruits after drying but the value of this variable in fresh fruits was nearly two times lower than in our study. Such considerable differences may be due to not only different measurement methods 
but also to the flesh color that is a cultivar specific feature. Changes in $a^{*}$ and $b^{*}$ variables were more strongly affected by the drying method, and were greater in fruits exposed to VMD than CD. Table 2 shows total color change range $\left(d \mathrm{E}^{*}\right)$ as compared with fresh fruits. $d \mathrm{E}^{*}$ was the lowest in FD samples, and the highest in the material dried in the microwave at high microwave power $(480 \mathrm{~W})$. The changes are associated with high sample temperature (Table 1) that causes formation of brown compounds in Maillard reaction [23]. Color changes were less pronounced in cv. 'PSI' than in 'GAL' and 'MSI', which was probably due to shorter drying time and lower sample temperature.

Table 2. Colour parameters as affected by different drying methods of jujube fruits.

\begin{tabular}{|c|c|c|c|c|c|}
\hline Cultivars & Drying Conditions & $d \mathrm{E}^{*}$ & $L^{*}$ & $a^{*}$ & $b^{*}$ \\
\hline \multirow{9}{*}{ ‘GAL' } & FRESH & & $81.78 \pm 0.68^{\dagger}$ & $-5.56 \pm 1.32$ & $19.59 \pm 0.87$ \\
\hline & FD & $2.95 \pm 2.45$ & $79.81 \pm 2.68$ & $-4.85 \pm 1.17$ & $17.5 \pm 1.58$ \\
\hline & VMD 480 & $34.42 \pm 4.2$ & $54.16 \pm 6.35$ & $10.87 \pm 1.73$ & $31.93 \pm 4.51$ \\
\hline & VMD 120 & $27.7 \pm 4.22$ & $58.86 \pm 4.8$ & $1.49 \pm 2.07$ & $33.46 \pm 1.43$ \\
\hline & VMD 480/120 & $19.67 \pm 1.82$ & $64.48 \pm 2.45 f$ & $-0.97 \pm 1.34$ & $27.75 \pm 2.11$ \\
\hline & CD-VMD 480/120 & $10.92 \pm 2.84$ & $72.4 \pm 2.7$ & $-1.71 \pm 3.62$ & $23.66 \pm 1.42$ \\
\hline & $\mathrm{CD} 50^{\circ} \mathrm{C}$ & $8.69 \pm 4.51$ & $75.21 \pm 5.13$ & $-0.95 \pm 1.27$ & $16.23 \pm 1.59$ \\
\hline & $\mathrm{CD} 60^{\circ} \mathrm{C}$ & $7.76 \pm 3$ & $74.43 \pm 3.35$ & $-3.2 \pm 1.25$ & $18.79 \pm 1.48$ \\
\hline & $\mathrm{CD} 70^{\circ} \mathrm{C}$ & $15.57 \pm 8.45$ & $67.37 \pm 8.3$ & $0.24 \pm 2.39$ & $18.45 \pm 3.81$ \\
\hline \multirow{9}{*}{ ‘MSI' } & FRESH & - & $79.35 \pm 1.49$ & $-7.19 \pm 3.03$ & $21.82 \pm 1.86$ \\
\hline & FD & $7.78 \pm 3.9$ & $71.86 \pm 3.9$ & $-6.34 \pm 1.37$ & $19.88 \pm 1.05$ \\
\hline & VMD 480 & $32.93 \pm 13.78$ & $49.98 \pm 14.65$ & $7.05 \pm 5.94$ & $26.19 \pm 13.53$ \\
\hline & VMD 120 & $20.1 \pm 2.76$ & $63.63 \pm 2.2 \mathrm{~cd}$ & $-1.69 \pm 1.43$ & $33.08 \pm 1.94$ \\
\hline & VMD 480/120 & $17.13 \pm 4.14$ & $65.72 \pm 4.34$ & $-0.79 \pm 2.52$ & $29.99 \pm 1.05$ \\
\hline & CD-VMD 480/120 & $17.29 \pm 2.9$ & $63.18 \pm 3.15$ & $-1.42 \pm 1.8$ & $23.88 \pm 1.81$ \\
\hline & $\mathrm{CD} 50^{\circ} \mathrm{C}$ & $16.46 \pm 2.26$ & $64.62 \pm 1.67$ & $-0.84 \pm 1.68$ & $18.11 \pm 3.01$ \\
\hline & $\mathrm{CD} 60^{\circ} \mathrm{C}$ & $18.4 \pm 3.81$ & $62.8 \pm 3.72$ & $0.07 \pm 1.43$ & $18.38 \pm 1.11$ \\
\hline & $\mathrm{CD} 70^{\circ} \mathrm{C}$ & $17.5 \pm 3.79$ & $64.36 \pm 4.68$ & $1.38 \pm 1.13$ & $18.93 \pm 0.72$ \\
\hline \multirow{9}{*}{ ‘PSI' } & FRESH & - & $78.88 \pm 0.29$ & $-6.19 \pm 0.52$ & $22.06 \pm 0.6$ \\
\hline & FD & $4.38 \pm 2.5$ & $75.16 \pm 3.53$ & $-5.31 \pm 0.92$ & $19.92 \pm 0.43$ \\
\hline & VMD 480 & $18.42 \pm 2.19$ & $63.28 \pm 1.95$ & $0.09 \pm 2.7$ & $29.58 \pm 1.81$ \\
\hline & VMD 120 & $20.82 \pm 1.09$ & $62.15 \pm 0.56$ & $-0.31 \pm 0.58$ & $32.97 \pm 2.58$ \\
\hline & VMD 480/120 & $17.21 \pm 2.53$ & $64.23 \pm 1.6$ & $-1.04 \pm 1.34$ & $29.48 \pm 2.19$ \\
\hline & CD-VMD 480/120 & $12.46 \pm 2.06$ & $68.48 \pm 2.2$ & $-0.29 \pm 2.36$ & $25.57 \pm 1.34$ \\
\hline & $\mathrm{CD} 50^{\circ} \mathrm{C}$ & $13.45 \pm 1.8$ & $66.4 \pm 1.5$ & $-1.26 \pm 1.39$ & $21.06 \pm 0.92$ \\
\hline & $\mathrm{CD} 60^{\circ} \mathrm{C}$ & $12.8 \pm 2.18$ & $66.9 \pm 1.88$ & $-1.74 \pm 2.23$ & $22.93 \pm 1.82$ \\
\hline & $\mathrm{CD} 70^{\circ} \mathrm{C}$ & $13.82 \pm 6.88$ & $66.43 \pm 6.83$ & $-0.2 \pm 1.77$ & $21.62 \pm 1.68$ \\
\hline \multicolumn{6}{|c|}{ Duncan's Multiple Range Test } \\
\hline \multirow{8}{*}{$\begin{array}{l}\text { Drying } \\
\text { treatment }\end{array}$} & FD & $5.52 c^{\dagger}$ & $75.52 \mathrm{e}$ & -5.50 & $19.09 \mathrm{a}$ \\
\hline & VMD 480 & $30.54 \mathrm{e}$ & $54.89 \mathrm{~d}$ & $6.61 d$ & $28.20 \mathrm{~b}$ \\
\hline & VMD 120 & 23.11d & $61.46 \mathrm{c}$ & $-0.10 a b$ & $33.25 \mathrm{~d}$ \\
\hline & VMD 480/120 & $18.25 b$ & $64.73 \mathrm{ac}$ & $-0.90 a b$ & $29.15 b$ \\
\hline & CD-VMD 480/120 & $13.91 \mathrm{a}$ & $67.95 \mathrm{ab}$ & $-1.12 \mathrm{ab}$ & $24.37 \mathrm{c}$ \\
\hline & CD 50 & $13.16 \mathrm{a}$ & $68.66 \mathrm{~b}$ & $-1.00 \mathrm{ab}$ & $18.45 a$ \\
\hline & CD 60 & $13.23 a$ & $67.96 \mathrm{ab}$ & $-1.62 \mathrm{a}$ & $20.06 a$ \\
\hline & CD 70 & $16.25 a$ & 65.67ab & $0.57 \mathrm{~b}$ & $19.66 a$ \\
\hline \multirow{3}{*}{ Cultivar } & 'GAL' & $16.51 \mathrm{ab}$ & $69.45 a$ & $-0.366 a$ & $23.07 \mathrm{a}$ \\
\hline & ${ }^{\prime} \mathrm{MSI}^{\prime}$ & $19.28 \mathrm{~b}$ & $64.47 \mathrm{~b}$ & $-0.795 a$ & $23.11 \mathrm{a}$ \\
\hline & ‘PSI' & $14.44 a$ & $67.74 a$ & $-1.716 a$ & $25.08 \mathrm{a}$ \\
\hline
\end{tabular}

${ }^{+}$mean value followed by the same letter \pm standard deviation SD values $\ddagger$ Values followed by the same letter, within the same column, are not significantly different $(p<0.05$, Duncan's multiple range test);-not detected; FD-freeze drying; CD-convective drying; VMD-vacuum-microwave drying; CD-VMDconvective-vacuum-microwave drying 


\subsection{Total Phenolic Compounds and Antioxidant Activity}

Control or reference values of total phenolic content (TPC) in the freeze-dried (FD) fruits were 3048, 3404, and $4454 \mathrm{mg} / 100 \mathrm{~g}$ d.w. for 'GAL', 'MSI', and 'PSI', respectively (Table 3). Similar initial values were reported by other researchers [16,27]. The main phenolic compounds in jujube fruits were flavan-3-ols (99\% of total polyphenolic compounds in 'GAL', 97\% in 'PSI', and 95\% in 'MSI'), with polymeric proanthocyanidins (PP) predominating and flavonols being the less abundant group.

Table 3. The influence of different methods and parameters on the total phenolic content $(\mathrm{mg} / 100 \mathrm{~g}$ $\mathrm{dm}$ ) and antioxidant capacity (ORAC, mmol TE/100 $\mathrm{g} \mathrm{dm}$ ) of jujube fruits.

\begin{tabular}{|c|c|c|c|}
\hline Cultivars & Drying Conditions & Total Polyphenols Content & ORAC \\
\hline \multirow{8}{*}{ ‘GAL' } & FD (control) & $3424 \pm 23^{\dagger}$ & $48.56 \pm 2.8$ \\
\hline & $\mathrm{CD} 50{ }^{\circ} \mathrm{C}$ & $3474 \pm 44$ & $38.79 \pm 4.6$ \\
\hline & $\mathrm{CD} 60^{\circ} \mathrm{C}$ & $2546 \pm 16$ & $37.51 \pm 2.1$ \\
\hline & $\mathrm{CD} 70^{\circ} \mathrm{C}$ & $1828 \pm 29$ & $32.99 \pm 2.2$ \\
\hline & VMD $120 \mathrm{~W}$ & $2271 \pm 31$ & $54.54 \pm 1.9$ \\
\hline & VMD $480 \mathrm{~W}$ & $2481 \pm 18$ & $44.59 \pm 2.6$ \\
\hline & VMD 480/120 W & $2964 \pm 25$ & $45.09 \pm 3.8$ \\
\hline & CD-VMD & $2881 \pm 27$ & $44.34 \pm 1.1$ \\
\hline \multirow{8}{*}{ 'MSI' } & FD (control) & $4287 \pm 31$ & $72.45 \pm 3.5$ \\
\hline & $\mathrm{CD} 50{ }^{\circ} \mathrm{C}$ & $2377 \pm 22$ & $54.83 \pm 2.7$ \\
\hline & $\mathrm{CD} 60^{\circ} \mathrm{C}$ & $2481 \pm 13$ & $34.14 \pm 2.2$ \\
\hline & $\mathrm{CD} 70^{\circ} \mathrm{C}$ & $1555 \pm 27$ & $20.81 \pm 1.8$ \\
\hline & VMD $120 \mathrm{~W}$ & $3237 \pm 31$ & $63.44 \pm 3.1$ \\
\hline & VMD $480 \mathrm{~W}$ & $2791 \pm 27$ & $57.58 \pm 2.1$ \\
\hline & VMD $480 / 120 \mathrm{~W}$ & $3920 \pm 24$ & $60.44 \pm 3.3$ \\
\hline & HaD-VMD & $3438 \pm 31$ & $58.13 \pm 1.4$ \\
\hline \multirow{8}{*}{ 'PSI' } & FD (control) & $5870 \pm 21$ & $66.67 \pm 2.7$ \\
\hline & $\mathrm{CD} 50^{\circ} \mathrm{C}$ & $5696 \pm 33$ & $64.71 \pm 1.9$ \\
\hline & $\mathrm{CD} 60^{\circ} \mathrm{C}$ & $4493 \pm 24$ & $59.87 \pm 2.5$ \\
\hline & $\mathrm{CD} 70^{\circ} \mathrm{C}$ & $3458 \pm 25$ & $35.86 \pm 3.1$ \\
\hline & VMD $120 \mathrm{~W}$ & $5343 \pm 35$ & $69.28 \pm 3.8$ \\
\hline & VMD $480 \mathrm{~W}$ & $5244 \pm 46$ & $77.90 \pm 4.1$ \\
\hline & VMD 480/120 W & $5076 \pm 52$ & $61.83 \pm 2.6$ \\
\hline & CD-VMD & $4432 \pm 14$ & $48.91 \pm 2.9$ \\
\hline \multicolumn{4}{|c|}{ Duncan's Multiple Range Test } \\
\hline \multirow{8}{*}{$\begin{array}{l}\text { Drying } \\
\text { treatment }\end{array}$} & FD (control) & $4527 \mathrm{a}^{\ddagger}$ & $62.56 a$ \\
\hline & $\mathrm{CD} 50^{\circ} \mathrm{C}$ & $3849 \mathrm{ab}$ & $52.78 \mathrm{abc}$ \\
\hline & $\mathrm{CD} 60^{\circ} \mathrm{C}$ & $3173 b c$ & $43.84 c$ \\
\hline & $\mathrm{CD} 70{ }^{\circ} \mathrm{C}$ & $2280 c$ & $29.89 d$ \\
\hline & VMD $120 \mathrm{~W}$ & $3617 \mathrm{ab}$ & $62.42 \mathrm{a}$ \\
\hline & VMD $480 \mathrm{~W}$ & $3505 \mathrm{ab}$ & $60.02 \mathrm{ab}$ \\
\hline & VMD $480 / 120 \mathrm{~W}$ & $3986 \mathrm{ab}$ & $55.78 \mathrm{ab}$ \\
\hline & HaD-VMD & $3583 a b$ & $50.46 \mathrm{bc}$ \\
\hline \multirow[t]{3}{*}{ Cultivar } & ‘GAL' & $2733 b$ & $43.30 c$ \\
\hline & ‘MSI' & $3010 b$ & $52.73 b$ \\
\hline & ‘PSI' & $4951 \mathrm{a}$ & $60.63 a$ \\
\hline
\end{tabular}

\footnotetext{
${ }^{\dagger}$ Values followed by the same letter \pm standard deviation ${ }^{\ddagger}$ Mean values for each processing followed by different letters are statistically different at $p<0.05$.
}

Drying method (FD, CD, VMD, and CD-VMD) and drying conditions significantly affected the contents of polyphenolic compounds. TPC content in all dried jujube samples followed the order FD $>>$ $\mathrm{VMD} \geq \mathrm{CD}-\mathrm{VMD}>\mathrm{CD}$. In terms of retaining TPC content the most efficient drying methods were FD $>$ $\mathrm{VMD}$ at $480 / 120 \mathrm{~W}>\mathrm{CD}-\mathrm{VMD}\left(50^{\circ} \mathrm{C}\right.$ and $\left.480 / 120 \mathrm{~W}\right)>\mathrm{VMD}$ at $120 \mathrm{~W}>\mathrm{VMD}$ at $480 \mathrm{~W}>\mathrm{CD}$ at $50{ }^{\circ} \mathrm{C}$. However, differences between the methods are not significant $(p>0.05)$. The study clearly indicates 
that TPC contents are retained more effectively $(p<0.05)$ when drying involves combined methods, such as pre-drying by $\mathrm{CD}$ and finished by VMD (CD-VMD) or even VMD with power adjustment along moisture content reduction, than the traditional hot-air drying (especially at 60 and $70^{\circ} \mathrm{C}$ ).

We noticed that an increase in VMD power from 120 to $480 \mathrm{~W}$ reduced TPC in jujube fruits from all three cultivars (Table 3$)$ but the trend was not significant $(p>0.05)$. Therefore, reducing the microwave power during VMD (from $480 \mathrm{~W}$ to $120 \mathrm{~W}$ ) to avoid sample overheating resulted in significantly higher content of TPC. This trend was confirmed for 'GAL' and 'MSI'.

As expected, the biggest changes in TPC were observed in CD samples that showed a clear dependency between the hot air temperature and polyphenol loss in all jujube cultivars (the higher the temperature-the lower TPC). Convective drying at $50{ }^{\circ} \mathrm{C}$ allowed for retaining maximum TPC.

Microwave heating inactivates degrading enzymes much faster than convective heating [4], yet a loss of phenolic compounds was measured. Gao et al. $[4,12]$ showed that oven heating at $70{ }^{\circ} \mathrm{C}$ rapidly inactivates polyphenol oxidases in jujube fruits. However, the enzymes may be active even earlier and degrade phenolic compounds at the initial stages of drying. Microwave-drying caused an insignificant $(5 \%)$ drop in phenolic compounds in jujube fruits [4].

Chen et al. [16] suggested that temperature is more important than time in drying of jujube fruits. They showed that an increase in the drying temperature, from 70 to $80^{\circ} \mathrm{C}$, significantly reduced TPC. Similar findings were reported for vacuum-dried aronia fruits [5]. The authors of the study found that combining microwave and vacuum drying and reducing the power or wattage of the microwaves at the final stage of the process may significantly reduce the product temperature and limit the loss of bioactive compounds, thus improving the product quality [5]. As a consequence, and theoretically, VMD should yield products with higher content of nutrients and aroma compounds than CD.

Antioxidant activity (Table 3 ) of jujube fruits, similarly as polyphenols content, were related by the drying methods. The highest values $(p<0.05)$ of ORAC were found in dried fruits of cvs. 'PSI' > 'MSI' > 'GAL', with values of 60.63, 52.73, $43.30 \mathrm{mmol}$ TE/100 g d.w., respectively. As prospective, the antioxidant activity was the highest in FD samples (72.45, 66.67 and $48.56 \mathrm{mmol}$ TE/100 g d.w., for cv. 'MSI', 'PSI' and 'GAL', respectively). VMD was the second most effective method at retaining such bioactive compounds as polyphenols and ORAC value, especially sample treated by at $120 \mathrm{~W}$. Considering the microwave power, the smallest loss was observed for combined (CD-VMD) method and power reduction from $480 \mathrm{~W}$ to $120 \mathrm{~W}$. High air temperature (especially 60 or $70{ }^{\circ} \mathrm{C}$ ) during dehydration process caused significantly $(p<0.05)$ degradation of biologically active compounds that may also exhibit antioxidant properties. Similarly, Wojdyło et al. [28] showed the greatest reduction of antioxidant activity at $70{ }^{\circ} \mathrm{C}(48 \%)$ while drying sour cherry fruits. As a conclusion, a high temperature causes faster degradation of the compounds responsible for the antioxidant activity.

\section{Materials and Methods}

\subsection{Reagents and Standards}

(-)-Epicatechin, (+)-catechin, quercetin, and kaempferol -3-O-glucoside and -3-O-rutinoside were purchased from Extrasynthese (Lyon, France). Ascorbic acid, trolox, phloroglucinol, acetonitrile and methanol for UPLC were purchased from Sigma-Aldrich (Steinheim, Germany).

\subsection{Plant Material and Sample Preparation}

Approximately $2 \mathrm{~kg}$ of jujube fruits (Z. jujube) from each cvs. as 'GAL', 'MSI', 'PSI', were manually hand harvested from 20-year-old trees (from 3 trees cultivars) a farm in the village of San Isidro province of Alicante, Spain (19 m above sea level; 38 $18^{\circ} 22,29^{\prime \prime} \mathrm{N} \times 0^{\circ} 51^{\prime} 36,138^{\prime \prime} \mathrm{W}$ ); Jujube fruits before drying were pitted and cut for pieces. 


\subsection{Drying Experiments}

Jujube samples approx. $60 \mathrm{~g}$, were subjected to four different drying methods, which was continued until the moisture content of samples equaled $0.05 \mathrm{~kg} / \mathrm{kg} \mathrm{dm}$ :

(i) Hot air drying (CD) was conducted using dryer designed and built at the Institute of Agricultural Engineering (Wrocław University of Environmental and Life Sciences, Wrocław, Poland) [3]. Air velocity was $1 \mathrm{~m} / \mathrm{s}$ and hot air temperatures during process were 50,60 and $70{ }^{\circ} \mathrm{C}$.

(ii) Vacuum-microwave drying (VMD) was carried out in dryer SM-200 (Plazmatronika S.A., Wroclaw, Poland) [3]. During VMD the microwave power was set to at $120 \mathrm{~W}, 480 \mathrm{~W}$ and 480/120 W (Microwave power was reduced to $120 \mathrm{~W}$ at the initial microwave power of $480 \mathrm{~W}$, when the maximum temperature of sample was higher than $75^{\circ} \mathrm{C}$ ). The pressure in the VMD chamber varied between 4 and $6 \mathrm{kPa}$.

(iii) Combined drying (CD-VMFD) consisted of hot-air pre-drying (CD) at a temperature of $60^{\circ} \mathrm{C}$, followed by VMFD at 480/120 W, the hot-air pre-drying time was $120 \mathrm{~min}$.

(iv) Freeze-drying (FD) was used as the control sample carried out used the dryer Alpha 1-4 LSC (Martin Christ GmbH, Osterode am Harz, Germany) during $24 \mathrm{~h}$. During FD the pressure was reduced to $0.960 \mathrm{kPa}$. The temperature of shelves and drying chamber were 26 and $-60^{\circ} \mathrm{C}$, respectively.

\subsection{Drying Confirmed Kinetics}

According to sample mass losses measured during drying was evaluated drying kinetics for convection and vacuum drying methods. The moisture ratio $M R$ was determined using the following equation [29]:

$$
M R=\frac{M}{M_{0}}
$$

where $M$ is the actual moisture content and $M_{0}$ is the initial moisture content.

The initial moisture contents of fresh jujube were $4.32,4.12$, and $4.72 \mathrm{~kg} / \mathrm{kg}$ dry matter $(\mathrm{dm})$ for 'GAL', 'MSI', and 'PSI', respectively.

The moisture content of dried samples was determined by drying the previously ground samples in a vacuum dryer (SPT-200, ZEAMiL Horyzont, Krakow, Poland) for $24 \mathrm{~h}$ at temperature $80{ }^{\circ} \mathrm{C}$ and pressure $300 \mathrm{~Pa}$.

\subsection{Energy Consumption}

The energy consumption during drying was calculated according to [9]. The energy efficiencies for $\mathrm{CD}, \mathrm{VMD}$ and $\mathrm{CD}-\mathrm{VMD}$ were determined as the ratio of energy necessary for evaporation of free water from the sample to the energy consumed while drying. The specific energy consumptions for $\mathrm{CD}, \mathrm{VMD}$ and CD-VMD were determined as the ratio of energy consumption to the initial mass of the sample expressed as $\mathrm{kJ} \cdot \mathrm{g}^{-1} \mathrm{fw}$ or as the ratio of energy consumption to the mass of water removed from the sample during drying expressed as $\mathrm{kJ} \cdot \mathrm{g}^{-1}$ water.

\subsection{Colour}

The colour was determined on the surface of samples from the flesh side with reference to the colour space, CIE $L^{*} a^{*} b^{*}$ system using a Minolta Chroma Meter CR-400 (Minolta Co., Ltd., Osaka, Japan). The total change in the colour ( $\mathrm{dE}$ ) was calculated following the equation as described by [30]. The measurements were done in five replicates.

\subsection{Determination of Total Phenolic Compounds (TPC) by UPLC-PDA-FL Method}

A sample for the analysis of polyphenols was prepared as described previously by Wojdyło et al. [20]. The sample for quantitative (UPLC-PDA-FL; Waters, Milford and Taunton, Massachusetts, USA) analysis of total polyphenols expresses as sum of flavonols (as sum of quercetin and keampferol 
derivatives)) and flavan-3-ols (as sum of monomers, dimers, polymeric procyanidins) were performed as described previously by Wojdyło et al. [14]. Prior to the measurements, the equipment was calibrated using a standard for flavonol compounds were used quercetin-3-O-glucoside (at 0,1 to $5 \mathrm{mg}$ ), and for flavan-3-ols were used (-)-epicatechin (at 0.1 to $5 \mathrm{mg}$ ). All measurements were repeated three times, and expressed as mean value as $\mathrm{mg} / 100 \mathrm{~g} \mathrm{dm}$.

\subsection{Determination of Antioxidant Activity}

The extraction of sample for the antioxidant analysis was prepared as described previously by Wojdyło et al. [20]. The ORAC assay was determined as previously described by Ou et al. [21] using a RF-5301 PC spectrofluorometer (Shimadzu, Kyoto, Japan). Results were expressed as mmol $\mathrm{TE} / 100 \mathrm{~g} \mathrm{dm}$.

\subsection{Statistical Analysis}

An ANOVA was performed using Statistica version 12.0 (StatSoft; Krakow, Poland), and means were separated by Duncan's multiple range test. All analyzes were performed duplicated and present as mean value \pm standard deviation. TableCurve 2D Windows v 5.01 (Jandel Scientific Software, San Jose, CA, USA) enabled mathematical modelling with the highest values of determination coefficient $\left(R^{2}\right)$ and the lowest values of root-mean-square error (RMSE).

\section{Conclusions}

The study identified fruits of cv. 'PSI' as the most appropriate for drying, despite the highest initial moisture content. Drying of this cultivar was the most efficient due to the shortest drying time and the lowest energy consumption. Furthermore, samples of cv. 'PSI' reached satisfactory level of dryness at the lowest temperature, which most effectively limited the loss of polyphenolic compounds and retained high antioxidant activity. 'PSI' fruits experienced also the smallest change in color $\left(d \mathrm{E}^{*}\right)$.

Hot air drying at low temperature $\left(\mathrm{CD} 50{ }^{\circ} \mathrm{C}\right.$ ) was the best method (except for control FD), considering the content of polyphenols, antioxidant activity and color parameters. However, it required a few times more energy than microwave and vacuum drying. VMD method, particularly at high power values $(480 \mathrm{~W})$ heated the samples to high temperature that adversely affected fruit color (browning) and degraded polyphenolic compounds. Therefore, the combined drying method seems to be the most effective, as it provides good quality dried jujube fruits and requires relatively low energy consumption.

Author Contributions: Conceptualization, A.A.C.-B., A.W., A.F. and F.H.; methodology, K.L., A.W.; formal analysis, K.L., A.W., P.N.; investigation; writing-original draft preparation, A.W., K.L. writing-review and editing, A.W.

Funding: Publication supported by Wroclaw Centre of Biotechnology, programme The Leading National Research Centre (KNOW) for years 2014-2018. The publication was the result of the activity of the research group: "Plants4food".

Conflicts of Interest: The authors declare no conflict of interest. The funders had no role in the design of the study; in the collection, analyses, or interpretation of data; in the writing of the manuscript, or in the decision to publish the results.

\section{References}

1. Ahrné, L.M.; Pereira, N.R.; Staack, N.; Floberg, P. Microwave convective drying of plant foods at constant and variable microwave power. Dry. Technol. 2007, 25, 1149-1153. [CrossRef]

2. Brewer, M.S. Natural Antioxidants: sources, compounds, mechanisms of action, and potential applications. Compr. Rev. Food Sci. Food Saf. 2011, 10, 221-247. [CrossRef]

3. Calín-Sánchez, Á.; Figiel, A.; Lech, K.; Szumny, A.; Martínez-Tomé, J.; Carbonell-Barrachina, Á.A. Dying methods affect the aroma of Origanum majorana L. analyzed by GC-MS and descriptive sensory analysis. Ind. Crops Prod. 2015, 74, 218-227. 
4. Gao, Q.-H.; Wu, C.-S.; Wang, M.; Xu, B.-N.; Du, L.-J. Effect of drying of jujubes (Ziziphus jujuba Mill.) on the contents of sugars, organic acids, $\alpha$-tocopherol, $\beta$-carotene, and phenolic compounds. J. Agric. Food Chem. 2012, 60, 9642-9648. [CrossRef] [PubMed]

5. Samoticha, J.; Wojdyło, A.; Lech, K. The influence of different the drying methods on chemical composition and antioxidant activity in chokeberries. LWT Food Sci. Technol. 2016, 66, 484-489. [CrossRef]

6. Wojdyło, A.; Figiel, A.; Oszmiański, J. Effect of drying methods with the application of vacuum microwaves on the bioactive compounds, color, and antioxidant activity of strawberry fruits. J. Agric. Food Chem. 2009, 57, 1337-1343. [CrossRef] [PubMed]

7. Giri, S.K.; Prasad, S. Drying kinetics and rehydration characteristics of microwave-vacuum and convective hot-air dried mushrooms. J. Food Eng. 2007, 78, 512-521. [CrossRef]

8. Calín-Sanchez, Á.; Figiel, A.; Szarycz, M.; Lech, K.; Nuncio-Jáuregui, N.; Carbonell-Barrachina, Á.A. Drying kinetics and energy consumption in the dehydration of pomegranate (Punica granatum L.) arils and rind. FABT 2014, 7, 2071-2083. [CrossRef]

9. Calín-Sánchez, Á.; Figiel, A.; Wojdyło, A.; Szarycz, M.; Carbonell-Barrachina, Á.A. Drying of garlic slices using convective pre-drying and vacuum-microwave finishing drying: kinetics, energy consumption, and quality studies. FABT 2014, 7, 398-408. [CrossRef]

10. Durance, T.D.; Wang, J.H. Energy consumption, density, and rehydration rate of vacuum microwave- and hot-air convection- dehydrated tomatoes. J. Food Sci. 2002, 67, 2212-2216. [CrossRef]

11. Andrés, A.; Bilbao, C.; Fito, P. Drying kinetics of apple cylinders under combined hot air-microwave dehydration. J. Food Eng. 2004, 63, 71-78. [CrossRef]

12. Gao, Q.-H.; Wu, C.-S.; Yu, J.-G.; Wang, M.; Ma, Y.-J.; Li, C.-L. Textural characteristic, antioxidant activity, sugar, organic acid, and phenolic profiles of 10 promising jujube (Ziziphus jujuba Mill.) selections. J. Food Sci. 2012, 77, C1218-C1225. [CrossRef] [PubMed]

13. Hernández, F.; Noguera-Artiaga, L.; Burló, F.; Wojdyło, A.; Carbonell-Barrachina, Á.A.; Legua, P. Physico-chemical, nutritional, and volatile composition and sensory profile of Spanish jujube (Ziziphus jujuba Mill.) fruits. J. Sci. Food Agric. 2016, 96, 2682-2691. [CrossRef] [PubMed]

14. Wojdyło, A.; Figiel, A.; Legua, P.; Lech, K.; Carbonell-Barrachina, Á.A.; Hernández, F. Chemical composition, antioxidant capacity, and sensory quality of dried jujube fruits as affected by cultivar and drying method. Food Chem. 2016, 207, 170-179. [CrossRef] [PubMed]

15. Gao, Q.-H.; Wu, C.-S.; Wang, M. The jujube (Ziziphus jujuba Mill.) fruit: a review of current knowledge of fruit composition and health benefits. J. Agric. Food Chem. 2013, 61, 3351-3363. [CrossRef]

16. Chen, Q.; Bi, J.; Wu, X.; Yi, J.; Zhou, L.; Zhou, Y. Drying kinetics and quality attributes of jujube (Zizyphus jujuba Miller) slices dried by hot-air and short- and medium-wave infrared radiation. LWT Food Sci. Technol. 2015, 64, 759-766. [CrossRef]

17. Calín-Sánchez, Á.; Kharaghani, A.; Lech, K.; Figiel, A.; Carbonell-Barrachina, A.A.; Tsotsas, E. Drying kinetics and microstructural and sensory properties of black chokeberry (Aronia melanocarpa) as affected by drying Method. FABT 2015, 8, 63-74.

18. Michalska, A.; Wojdyło, A.; Lech, K.; Łysiak, G.P.; Figiel, A. Physicochemical properties of whole fruit plum powders obtained using different drying technologies. Food Chem. 2016, 207, 223-232. [CrossRef]

19. Pillai, M.G. Thin layer drying kinetics, characteristics and modeling of plaster of Paris. Chem. Eng. Res. Des. 2013, 91, 1018-1027. [CrossRef]

20. Cano-Lamadrid, M.; Lech, K.; Michalska, A.; Wasilewska, M.; Figiel, A.; Wojdyło, A.; Carbonell-Barrachina, Á.A. Influence of osmotic dehydration pre-treatment and combined drying method on physico-chemical and sensory properties of pomegranate arils, cultivar Mollar de Elche. Food Chem. 2017, 232, 306-315. [CrossRef]

21. Sharma, G.P.; Prasad, S. Drying of garlic (Allium sativum) cloves by microwave-hot air combination. J. Food Eng. 2001, 50, 99-105. [CrossRef]

22. Held, C.; Sadowski, G. Compatible solutes: Thermodynamic properties relevant for effective protection against osmotic stress. Fluid Phase Equilibria 2016, 407, 224-235. [CrossRef]

23. Motevali, A.; Minaei, S.; Khoshtagaza, M.H. Evaluation of energy consumption in different drying methods. Energy Convers. Manag. 2011, 52, 1192-1199. [CrossRef]

24. Soysal, Y.; Öztekin, S.; Eren, Ö. Microwave Drying of Parsley: Modelling, Kinetics, and Energy Aspects. Biosyst. Eng. 2006, 93, 403-413. [CrossRef] 
25. Jiang, N.; Liu, C.; Li, D.; Zhang, Z.; Liu, C.; Wang, D.; Zhang, M. Evaluation of freeze drying combined with microwave vacuum drying for functional okra snacks: Antioxidant properties, sensory quality, and energy consumption. LWT Food Sci. Technol. 2017, 82, 216-226. [CrossRef]

26. Sharma, G.P.; Prasad, S. Specific energy consumption in microwave drying of garlic cloves. Energy 2006, 31, 1921-1926. [CrossRef]

27. Choi, S.-H.; Ahn, J.-B.; Kozukue, N.; Levin, C.E.; Friedman, M. Distribution of free amino acids, flavonoids, total phenolics, and antioxidative activities of Jujube (Ziziphus jujuba) fruits and seeds harvested from plants grown in Korea. J. Agric. Food Chem. 2011, 59, 6594-6604. [CrossRef]

28. Wojdyło, A.; Figiel, A.; Lech, K.; Nowicka, P.; Oszmiański, J. Effect of convective and vacuum-microwave drying on the bioactive compounds, color, and antioxidant capacity of sour cherries. FABT 2014, 7, 829-841. [CrossRef]

29. Lech, K.; Figiel, A.; Wojdyło, A.; Korzeniowska, M.; Serowik, M.; Szarycz, M. Drying kinetics and bioactivity of beetroot slices pretreated in concentrated chokeberry juice and dried with vacuum microwaves. Dry. Technol. 2015, 33, 1644-1653. [CrossRef]

30. Buvé, C.; Kebede, B.T.; De Batselier, C.; Carrillo, C.; Pham, H.T.T.; Hendrickx, M.; Van Loey, A. Kinetics of colour changes in pasteurised strawberry juice during storage. J. Food Eng. 2018, 216, $42-51$.

Sample Availability: Sample Availability: Samples of the compounds of dried jujube fruits are available from the authors.

(C) 2019 by the authors. Licensee MDPI, Basel, Switzerland. This article is an open access article distributed under the terms and conditions of the Creative Commons Attribution (CC BY) license (http://creativecommons.org/licenses/by/4.0/). 